\title{
Asymptotic behavior of random coupled Ginzburg-Landau equation driven by colored noise on unbounded domains
}

\author{
Zhang Chen', Lingyu Li ${ }^{1 *}$ and Dandan Yang
}

"Correspondence:

lyli@mail.sdu.edu.cn

'School of Mathematics, Shandong

University, 250100 Jinan, P.R. China

\begin{abstract}
In this paper, a random coupled Ginzburg-Landau equation driven by colored noise on unbounded domains is considered, in which the nonlinear term satisfies a local Lipschitz condition. It is shown that the random attractor of such a coupled Ginzburg-Landau equation is a singleton set, and the components of solutions are very close when the coupling parameter becomes large enough.
\end{abstract}

Keywords: Random coupled Ginzburg-Landau equation; Colored noise; Random attractor; Singleton set

\section{Introduction}

Synchronization phenomenon, which was discovered in physics, biology, and social science areas [1-3], has been paid more attention due to its extensive applications in secure communications, modeling brain activity, and optimization of nonlinear system performance $[4,5]$. Synchronization of deterministic coupled dissipative systems has been investigated [6-9].

Since noise is omnipresent in the real world, random perturbation is an important factor worthy of being considered in synchronization. In 2005, Caraballo and Kloeden [10] investigated the persistence of synchronization under additive noise and the asymptotic behavior of the coupled systems as the coupling parameter becomes arbitrarily large. In [11], the convergence rate of synchronization for stochastic ordinary differential equations with the vector-valued additive noise was obtained. Moreover, it is investigated for synchronization of a coupled sine-Gordon wave model on bounded domains with Neumann boundary conditions perturbed by additive white noise by the quasi-stability method [12].

For the case of linear multiplicative noises, the synchronization of Stratonovich stochastic differential equations was investigated in [13] by transforming it to random ordinary differential equations. Recently, synchronization for stochastic differential equations with additive noise and linear multiplicative noise was investigated in [14] by transforming it to random ordinary differential equations using the theory of Imkeller and Schmalfuss. However, the methods in the above references cannot deal with synchronization for systems with nonlinear noise. Li and Liu [15] proved the synchronization result for stochas-

(c) The Author(s) 2021. This article is licensed under a Creative Commons Attribution 4.0 International License, which permits use, sharing, adaptation, distribution and reproduction in any medium or format, as long as you give appropriate credit to the original author(s) and the source, provide a link to the Creative Commons licence, and indicate if changes were made. The images or other third party material in this article are included in the article's Creative Commons licence, unless indicated otherwise in a credit line to the material. If material is not included in the article's Creative Commons licence and your intended use is not permitted by statutory regulation or exceeds the permitted use, you will need to obtain permission directly from the copyright holder. To view a copy of this licence, visit http://creativecommons.org/licenses/by/4.0/. 
tic differential equations with general nonlinear multiplicative noise in the mean square sense.

It is worth mentioning that the nonlinear terms in the above literature are satisfied with one-sided dissipative Lipschitz conditions or global Lipschitz conditions. When the nonlinear term is satisfied with local Lipschitz condition, Caraballo, Chueshov, and Kloeden [16] studied random attractor and synchronization for a stochastic reaction-diffusion system with additive space-time noise on a thin bounded domain.

Motivated by the above literature, in this paper, we consider the random coupled complex Ginzburg-Landau equations driven by multiplicative colored noise on unbounded domains:

$$
\left\{\begin{array}{l}
\frac{\partial u^{\varepsilon}}{\partial t}-(1+i \lambda) \Delta u^{\varepsilon}=-\rho_{1} u^{\varepsilon}+f\left(u^{\varepsilon}\right)+\varepsilon\left(v^{\varepsilon}-u^{\varepsilon}\right)+u^{\varepsilon} \mathcal{G}_{\delta}\left(\theta_{t} \omega\right), \\
\frac{\partial \nu^{\varepsilon}}{\partial t}-(1+i \lambda) \Delta v^{\varepsilon}=-\rho_{2} v^{\varepsilon}+f\left(v^{\varepsilon}\right)+\varepsilon\left(u^{\varepsilon}-v^{\varepsilon}\right)+v^{\varepsilon} \mathcal{G}_{\delta}\left(\theta_{t} \omega\right), \\
u^{\varepsilon}(\tau, x)=u_{\tau}(x), \quad v^{\varepsilon}(\tau, x)=v_{\tau}(x)
\end{array}\right.
$$

where $u^{\varepsilon}(t, x), v^{\varepsilon}(t, x)$ are unknown complex-valued functions, $t \geq \tau, x \in \mathbb{R}, i$ is the imaginary unit, $\lambda, \mu \in \mathbb{R}, \rho_{1}, \rho_{2}>0$, the nonlinear term $f(u)=-(1+i \mu)|u|^{2} u$ is a complexvalued function, $\varepsilon>0$ is a coupling parameter, and $\mathcal{G}_{\delta}\left(\theta_{t} \omega\right)$ is the colored noise introduced in $[17,18]$ and the unique stationary solution of the stochastic differential equation $\mathrm{d} \mathcal{G}_{\delta}+\frac{1}{\delta} \mathcal{G}_{\delta} \mathrm{d} t=\frac{1}{\delta} \mathrm{d} W$. It is worth noting that the nonlinear term $f$ in (1) does not satisfy global Lipschitz conditions such as [12] or one-sided Lipschitz conditions such as [13, 14]. Moreover, different from the case of bounded domains in [12,16], Sobolev embedding on unbounded domains is noncompact. In [19], the authors investigated random attractor for nonautonomous random Ginzburg-Landau equation driven by nonlinear colored noise on unbounded domains by the tail-estimates method and the properties of the colored noise. In this paper, we further prove that the solutions of (1) converge pathwise to each other and the random attractor set is a singleton set in Sect. 3. Moreover, it is also proved that the solution $\left(u^{\varepsilon}, v^{\varepsilon}\right)$ of coupled system (1) satisfies $\lim _{\varepsilon \rightarrow+\infty}\left\|u^{\varepsilon}(t)-v^{\varepsilon}(t)\right\|^{2}=0$ uniformly on any bounded time-interval. In addition, one can refer to [20] for random attractor of fractional Ginzburg-Landau equation driven by colored noise on bounded domains and to [21] for random attractor of coupled fractional Ginzburg-Landau equation.

Throughout this paper, let $\|\cdot\|$ and $(\cdot, \cdot)$ denote the norm and the inner product of $L^{2}(\mathbb{R})$, respectively. The Sobolev space $H^{k}(\mathbb{R})(k \in \mathbb{N})$ consists of all $u \in L^{2}(\mathbb{R})$ whose weak derivatives up to order $k$ belong to $L^{2}(\mathbb{R})$ as well, which is a separable Banach space with the norm $\|u\|_{H^{k}(\mathbb{R})}:=\left(\sum_{|\alpha| \leq k} \int_{\mathbb{R}}\left|D^{\alpha} u(x)\right|^{2} \mathrm{~d} x\right)^{\frac{1}{2}}$. Denote $|\xi|_{H^{k}(\mathbb{R})}^{2}:=\|u\|_{H^{k}(\mathbb{R})}^{2}+\|v\|_{H^{k}(\mathbb{R})^{\prime}}^{2}$, $|\xi|_{L^{k}(\mathbb{R})}^{k}:=\|u\|_{L^{k}(\mathbb{R})}^{k}+\|v\|_{L^{k}(\mathbb{R})}^{k}$, where $\xi=(u, v)^{T}$.

\section{Preliminaries}

In this section, we recall some properties about the colored noise, which are useful for the proof of the main results. There exists a $\theta_{t}$-invariant subset of full measure (see [22]), which is still denoted by $\Omega$, such that, for all $\omega \in \Omega$,

$$
\lim _{t \rightarrow \pm \infty} \frac{\omega(t)}{t}=0
$$


For every $\omega \in \Omega$ and $\delta \in(0,1]$, we write

$$
\mathcal{G}_{\delta}(\omega)=\frac{1}{\delta} \int_{-\infty}^{0} e^{\frac{s}{\delta}} \mathrm{d} W=-\frac{1}{\delta^{2}} \int_{-\infty}^{0} e^{\frac{s}{\delta}} \omega(s) \mathrm{d} s
$$

Then $\mathcal{G}_{\delta}\left(\theta_{t} \omega\right)$ is the so-called Ornstein-Uhlenbeck process.

Lemma 2.1 ([23]) (1) For every $\omega \in \Omega$, the mapping $t \mapsto \mathcal{G}_{\delta}\left(\theta_{t} \omega\right)$ is continuous, and for every $0<\delta \leq 1$,

$$
\lim _{t \rightarrow \pm \infty} \frac{\left|\mathcal{G}_{\delta}\left(\theta_{t} \omega\right)\right|}{t}=0
$$

(2) For every $\omega \in \Omega$,

$$
\lim _{t \rightarrow \pm \infty} \frac{1}{t} \int_{0}^{t} \mathcal{G}_{\delta}\left(\theta_{s} \omega\right) \mathrm{d} s=0 \quad \text { uniformly for } 0<\delta \leq 1
$$

Lemma 2.2 ([24]) Let $\tau \in \mathbb{R}, \omega \in \Omega$ and $T>0$. Then there exist $\delta_{0}=\delta_{0}(\tau, \omega, T)>0$ and $M=M(\tau, \omega, T)>0$ such that, for all $0<\delta<\delta_{0}$ and $t \in[\tau, \tau+T]$,

$$
\left|\int_{0}^{t} \mathcal{G}_{\delta}\left(\theta_{s} \omega\right) \mathrm{d} s\right| \leq M
$$

\section{The asymptotic behavior of the coupled system}

In this section, we consider the random coupled Ginzburg-Landau equation driven by multiplicative colored noise (1). Our goal is to prove that the random attractor set of coupled system (1) is a singleton set for any fixed $\varepsilon>0$. Moreover, the solution $u^{\varepsilon}(t) \rightarrow v^{\varepsilon}(t)$ uniformly for $t$ on any bounded time-interval as the coupling parameter $\varepsilon$ tends to infinity.

Set

$$
\xi^{\varepsilon}=\left[\begin{array}{c}
u^{\varepsilon} \\
v^{\varepsilon}
\end{array}\right], \quad F\left(\xi^{\varepsilon}\right)=\left[\begin{array}{c}
-\rho_{1} u^{\varepsilon}+f\left(u^{\varepsilon}\right) \\
-\rho_{2} v^{\varepsilon}+f\left(v^{\varepsilon}\right)
\end{array}\right], \quad B=\left[\begin{array}{cc}
-1 & 1 \\
1 & -1
\end{array}\right]
$$

Then (1) is rewritten as

$$
\frac{\partial \xi^{\varepsilon}}{\partial t}-(1+i \lambda) \Delta \xi^{\varepsilon}=F\left(\xi^{\varepsilon}\right)+\varepsilon B \xi^{\varepsilon}+\xi^{\varepsilon} \mathcal{G}_{\delta}\left(\theta_{t} \omega\right)
$$

with the initial datum $\left(u_{\tau}, v_{\tau}\right)^{T}$.

Similar to the discussion in [19], we can obtain that system (2) has a unique solution $\xi^{\varepsilon} \in C\left([\tau, \infty) ; L^{2}(\mathbb{R}) \times L^{2}(\mathbb{R})\right) \cap L_{\text {loc }}^{2}\left([\tau, \infty) ; H^{1}(\mathbb{R}) \times H^{1}(\mathbb{R})\right)$, and $\xi^{\varepsilon} \in L_{\text {loc }}^{4}\left([\tau, \infty) ; L^{4}(\mathbb{R}) \times\right.$ $\left.L^{4}(\mathbb{R})\right)$. What is more, system (2) has a unique random attractor. In what follows, we show that the random attractor set is a singleton set.

Theorem 3.1 For any $\rho_{1}, \rho_{2}>0$, the random attractor sets of coupled system (2) are singleton sets for any given $\varepsilon>0$. 
Proof Let $\xi^{\varepsilon}=\left(u_{1}^{\varepsilon}, v_{1}^{\varepsilon}\right)^{T}$ and $\eta^{\varepsilon}=\left(u_{2}^{\varepsilon}, v_{2}^{\varepsilon}\right)^{T}$ be the solutions of (2) with the initial data $\xi_{\tau}=$ $\left(u_{1, \tau}, v_{1, \tau}\right)^{T}$ and $\eta_{\tau}=\left(u_{2, \tau}, v_{2, \tau}\right)^{T}$, respectively. Then we have

$$
\begin{aligned}
\frac{\mathrm{d}}{\mathrm{d} t}\left|\xi^{\varepsilon}-\eta^{\varepsilon}\right|^{2}+2\left|\nabla\left(\xi^{\varepsilon}-\eta^{\varepsilon}\right)\right|^{2} & \\
= & 2 \operatorname{Re}\left\langle F\left(\xi^{\varepsilon}\right)-F\left(\eta^{\varepsilon}\right), \xi^{\varepsilon}-\eta^{\varepsilon}\right\rangle+2 \operatorname{Re}\left\langle\varepsilon B\left(\xi^{\varepsilon}-\eta^{\varepsilon}\right), \xi^{\varepsilon}-\eta^{\varepsilon}\right\rangle \\
& +2 \mathcal{G}_{\delta}\left(\theta_{t} \omega\right)\left|\xi^{\varepsilon}-\eta^{\varepsilon}\right|^{2} .
\end{aligned}
$$

For the first term on the right-hand side of (3), it follows from the Hölder inequality and the Young inequality that

$$
\begin{aligned}
& \operatorname{Re}\left\langle F\left(\xi^{\varepsilon}\right)-F\left(\eta^{\varepsilon}\right), \xi^{\varepsilon}-\eta^{\varepsilon}\right\rangle \\
& \leq- \rho_{1}\left\|u_{1}^{\varepsilon}-u_{2}^{\varepsilon}\right\|^{2}-\rho_{2}\left\|v_{1}^{\varepsilon}-v_{2}^{\varepsilon}\right\|^{2}+\min \left\{\frac{\rho_{1}}{2}, \frac{\rho_{2}}{2}, 1\right\}\left\|u_{1}^{\varepsilon}-u_{2}^{\varepsilon}\right\|_{H^{1}(\mathbb{R})}^{2} \\
&+\min \left\{\frac{\rho_{1}}{2}, \frac{\rho_{2}}{2}, 1\right\}\left\|v_{1}^{\varepsilon}-v_{2}^{\varepsilon}\right\|_{H^{1}(\mathbb{R})}^{2} \\
&+\frac{3}{2^{\frac{4}{3}}}\left(1+2 \sqrt{2} \sqrt{1+\mu^{2}}\right)^{\frac{4}{3}}\left(\min \left\{\frac{\rho_{1}}{2}, \frac{\rho_{2}}{2}, 1\right\}\right)^{-\frac{1}{3}} \\
&+\left[\left(\left\|u_{1}^{\varepsilon}\right\|_{L^{4}(\mathbb{R})}^{\frac{8}{3}}+\left\|u_{2}^{\varepsilon}\right\|_{L^{4}(\mathbb{R})}^{\frac{8}{3}}\right)\left\|u_{1}^{\varepsilon}-u_{2}^{\varepsilon}\right\|^{2}\right. \\
&\left.+\left(\left\|v_{1}^{\varepsilon}\right\|_{L^{4}(\mathbb{R})}^{\frac{8}{3}}+\left\|v_{2}^{\varepsilon}\right\|_{L^{4}(\mathbb{R})}^{\frac{8}{3}}\right)\left\|v_{1}^{\varepsilon}-v_{2}^{\varepsilon}\right\|^{2}\right] \\
& \leq \min \left\{\rho_{1}, \rho_{2}\right\}\left|\xi^{\varepsilon}-\eta^{\varepsilon}\right|^{2}+\min \left\{\frac{\rho_{1}}{2}, \frac{\rho_{2}}{2}, 1\right\}\left|\xi^{\varepsilon}-\eta^{\varepsilon}\right|_{H^{1}(\mathbb{R})}^{2} \\
&+C\left(\xi^{\varepsilon}, \eta^{\varepsilon}\right)\left|\xi^{\varepsilon}-\eta^{\varepsilon}\right|^{2},
\end{aligned}
$$

where

$$
\begin{aligned}
& C\left(\xi^{\varepsilon}, \eta^{\varepsilon}\right)=a\left[\left|\xi^{\varepsilon}\right|_{L^{4}(\mathbb{R})}^{\frac{8}{3}}+\left|\eta^{\varepsilon}\right|_{L^{4}(\mathbb{R})}^{\frac{8}{3}}\right], \\
& a=\frac{3}{2^{\frac{4}{3}}}\left(1+2 \sqrt{2} \sqrt{1+\mu^{2}}\right)^{\frac{4}{3}}\left(\min \left\{\frac{\rho_{1}}{2}, \frac{\rho_{2}}{2}, 1\right\}\right)^{-\frac{1}{3}} .
\end{aligned}
$$

Then, together with (3), we have

$$
\begin{aligned}
& \frac{\mathrm{d}}{\mathrm{d} t}\left|\xi^{\varepsilon}-\eta^{\varepsilon}\right|^{2} \\
& \quad \leq-\min \left\{\rho_{1}, \rho_{2}\right\}\left|\xi^{\varepsilon}-\eta^{\varepsilon}\right|^{2}+2 C\left(\xi^{\varepsilon}, \eta^{\varepsilon}\right)\left|\xi^{\varepsilon}-\eta^{\varepsilon}\right|^{2}+2 \mathcal{G}_{\delta}\left(\theta_{t} \omega\right)\left|\xi^{\varepsilon}-\eta^{\varepsilon}\right|^{2}
\end{aligned}
$$

From the Gronwall inequality, we can obtain

$$
\left|\xi^{\varepsilon}-\eta^{\varepsilon}\right|^{2} \leq e^{-\min \left\{\rho_{1}, \rho_{2}\right\}(t-\tau)+2 \int_{\tau}^{t} \mathcal{G}_{\delta}\left(\theta_{s} \omega\right) \mathrm{d} s+2 \int_{\tau}^{t} C\left(\xi^{\varepsilon}, \eta^{\varepsilon}\right) \mathrm{d} s}\left|\xi_{\tau}-\eta_{\tau}\right|^{2}
$$


Since

$$
\begin{aligned}
& \frac{\mathrm{d}}{\mathrm{d} t}\left|\xi^{\varepsilon}\right|^{2}+2\left|\nabla\left(\xi^{\varepsilon}\right)\right|^{2} \\
& \quad=2 \operatorname{Re}\left\langle F\left(\xi^{\varepsilon}\right), \xi^{\varepsilon}\right\rangle+2 \operatorname{Re}\left\langle\varepsilon B \xi^{\varepsilon}, \xi^{\varepsilon}\right\rangle+2 \mathcal{G}_{\delta}\left(\theta_{t} \omega\right)\left|\xi^{\varepsilon}\right|^{2} \\
& \quad \leq-2 \rho_{1}\left\|u_{1}^{\varepsilon}\right\|^{2}-2 \rho_{2}\left\|v_{1}^{\varepsilon}\right\|^{2}-2\left\|u_{1}^{\varepsilon}\right\|_{L^{4}(\mathbb{R})}^{4}-2\left\|v_{1}^{\varepsilon}\right\|_{L^{4}(\mathbb{R})}^{4}+2 \mathcal{G}_{\delta}\left(\theta_{t} \omega\right)\left|\xi^{\varepsilon}\right|^{2} \\
& \quad \leq-2 \min \left\{\rho_{1}, \rho_{2}\right\}\left|\xi^{\varepsilon}\right|^{2}-2\left\|u_{1}^{\varepsilon}\right\|_{L^{4}(\mathbb{R})}^{4}-2\left\|v_{1}^{\varepsilon}\right\|_{L^{4}(\mathbb{R})}^{4}+2 \mathcal{G}_{\delta}\left(\theta_{t} \omega\right)\left|\xi^{\varepsilon}\right|^{2}
\end{aligned}
$$

we have

$$
\begin{gathered}
\frac{\mathrm{d}}{\mathrm{d} t}\left|\xi^{\varepsilon}\right|^{2}+2\left|\nabla\left(\xi^{\varepsilon}\right)\right|^{2}+2\left\|u_{1}^{\varepsilon}\right\|_{L^{4}(\mathbb{R})}^{4}+2\left\|v_{1}^{\varepsilon}\right\|_{L^{4}(\mathbb{R})}^{4} \\
\leq-2 \min \left\{\rho_{1}, \rho_{2}\right\}\left|\xi^{\varepsilon}\right|^{2}+2 \mathcal{G}_{\delta}\left(\theta_{t} \omega\right)\left|\xi^{\varepsilon}\right|^{2}
\end{gathered}
$$

By the Gronwall inequality, one can obtain

$$
\left|\xi^{\varepsilon}\right|^{2} \leq e^{2 \int_{\tau}^{t}\left[-\min \left\{\rho_{1}, \rho_{2}\right\}+\mathcal{G}_{\delta}\left(\theta_{s} \omega\right)\right] \mathrm{d} s}\left|\xi_{\tau}\right|^{2} .
$$

From (5) and (6), it follows that

$$
\begin{aligned}
& \int_{\tau}^{t}\left\|u_{1}^{\varepsilon}\right\|_{L^{4}(\mathbb{R})}^{4} \mathrm{~d} s+\int_{\tau}^{t}\left\|v_{1}^{\varepsilon}\right\|_{L^{4}(\mathbb{R})}^{4} \mathrm{~d} s \\
& \quad \leq \frac{1}{2}\left|\xi_{\tau}\right|^{2}+\int_{\tau}^{t} \mathcal{G}_{\delta}\left(\theta_{s} \omega\right)\left|\xi^{\varepsilon}\right|^{2} \mathrm{~d} s \\
& \quad \leq \frac{1}{2}\left|\xi_{\tau}\right|^{2}+\int_{\tau}^{t}\left|\mathcal{G}_{\delta}\left(\theta_{s} \omega\right)\right| e^{2 \int_{\tau}^{s}\left[-\min \left\{\rho_{1}, \rho_{2}\right\}+\mathcal{G}_{\delta}\left(\theta_{r} \omega\right)\right] \mathrm{d} r}\left|\xi_{\tau}\right|^{2} \mathrm{~d} s
\end{aligned}
$$

By Lemma 2.1, there exists $T(\omega)$ such that, for all $t>T(\omega), \int_{\tau}^{t} \mathcal{G}_{\delta}\left(\theta_{s} \omega\right) \mathrm{d} s \leq \frac{\min \left\{\rho_{1}, \rho_{2}\right\}}{4}(t-\tau)$ and $\left|\mathcal{G}_{\delta}\left(\theta_{t} \omega\right)\right| \leq t$. In addition, by Lemma 2.2, there exists $M(\omega)$ such that $\int_{\tau}^{T(\omega)}\left|\mathcal{G}_{\delta}\left(\theta_{t} \omega\right)\right| \mathrm{d} t \leq M(\omega)$. Then, together with (7), we obtain

$$
\begin{aligned}
\int_{\tau}^{t}\left\|u_{1}^{\varepsilon}\right\|_{L^{4}(\mathbb{R})}^{4} \mathrm{~d} s+\int_{\tau}^{t}\left\|v_{1}^{\varepsilon}\right\|_{L^{4}(\mathbb{R})}^{4} \mathrm{~d} s \\
\leq \frac{1}{2}\left|\xi_{\tau}\right|^{2}+\left|\xi_{\tau}\right|^{2}\left[\int_{\tau}^{T(\omega)}\left|\mathcal{G}_{\delta}\left(\theta_{s} \omega\right)\right| e^{2 \int_{\tau}^{s}\left[-\min \left\{\rho_{1}, \rho_{2}\right\}+\mathcal{G}_{\delta}\left(\theta_{r} \omega\right)\right] \mathrm{d} r} \mathrm{~d} s\right. \\
\left.\quad+\int_{T(\omega)}^{t}\left|\mathcal{G}_{\delta}\left(\theta_{s} \omega\right)\right| e^{2 \int_{\tau}^{s}\left[-\min \left\{\rho_{1}, \rho_{2}\right\}+\mathcal{G}_{\delta}\left(\theta_{r} \omega\right)\right] \mathrm{d} r} \mathrm{~d} s\right] \\
\leq M^{\prime}(\omega)\left|\xi_{\tau}\right|^{2}+\left|\xi_{\tau}\right|^{2} \int_{T(\omega)}^{t} s e^{-\frac{3}{2} \min \left\{\rho_{1}, \rho_{2}\right\}(s-\tau)} \mathrm{d} s,
\end{aligned}
$$

where $M^{\prime}(\omega)=\int_{\tau}^{T(\omega)}\left|\mathcal{G}_{\delta}\left(\theta_{s} \omega\right)\right| e^{2 \int_{\tau}^{s}\left[-\min \left\{\rho_{1}, \rho_{2}\right\}+\mathcal{G}_{\delta}\left(\theta_{r} \omega\right)\right] \mathrm{d} r} \mathrm{~d} s+\frac{1}{2}$.

Similarly, we have

$$
\begin{aligned}
& \int_{\tau}^{t}\left\|u_{2}^{\varepsilon}\right\|_{L^{4}(\mathbb{R})}^{4} \mathrm{~d} s+\int_{\tau}^{t}\left\|v_{2}^{\varepsilon}\right\|_{L^{4}(\mathbb{R})}^{4} \mathrm{~d} s \\
& \leq M^{\prime}(\omega)\left|\eta_{\tau}\right|^{2}+\left|\eta_{\tau}\right|^{2} \int_{T(\omega)}^{t} s e^{-\frac{3}{2} \min \left\{\rho_{1}, \rho_{2}\right\}(s-\tau)} \mathrm{d} s
\end{aligned}
$$


From (8), (9), and the Hölder inequality, it follows that

$$
\begin{aligned}
\int_{\tau}^{t} C & \left(\xi^{\varepsilon}, \eta^{\varepsilon}\right) \mathrm{d} s \\
\leq & a\left[\left(\int_{\tau}^{t}\left|\xi^{\varepsilon}\right|_{L^{4}(\mathbb{R})}^{4} \mathrm{~d} s\right)^{\frac{2}{3}}+\left(\int_{\tau}^{t}\left|\eta^{\varepsilon}\right|_{L^{4}(\mathbb{R})}^{4} \mathrm{~d} s\right)^{\frac{2}{3}}\right](t-\tau)^{\frac{1}{3}} \\
\leq & a\left[\left(M^{\prime}(\omega)\left|\xi_{\tau}\right|^{2}+\left|\xi_{\tau}\right|^{2} \int_{T(\omega)}^{t} s e^{-\frac{3}{2} \min \left\{\rho_{1}, \rho_{2}\right\}(s-\tau)} \mathrm{d} s\right)^{\frac{2}{3}}\right. \\
+ & \left.\left(M^{\prime}(\omega)\left|\eta_{\tau}\right|^{2}+\left|\eta_{\tau}\right|^{2} \int_{T(\omega)}^{t} s e^{-\frac{3}{2} \min \left\{\rho_{1}, \rho_{2}\right\}(s-\tau)} \mathrm{d} s\right)^{\frac{2}{3}}\right](t-\tau)^{\frac{1}{3}} \\
\leq & a\left[\left(M^{\prime}(\omega)\left|\xi_{\tau}\right|^{2}+\left|\xi_{\tau}\right|^{2} \int_{T(\omega)}^{+\infty} s e^{-\frac{3}{2} \min \left\{\rho_{1}, \rho_{2}\right\}(s-\tau)} \mathrm{d} s\right)^{\frac{2}{3}}\right. \\
& \left.+\left(M^{\prime}(\omega)\left|\eta_{\tau}\right|^{2}+\left|\eta_{\tau}\right|^{2} \int_{T(\omega)}^{+\infty} s e^{-\frac{3}{2} \min \left\{\rho_{1}, \rho_{2}\right\}(s-\tau)} \mathrm{d} s\right)^{\frac{2}{3}}\right](t-\tau)^{\frac{1}{3}} \\
:= & C\left(\omega, \xi_{\tau}, \eta_{\tau}\right)(t-\tau)^{\frac{1}{3}},
\end{aligned}
$$

which together with (4) implies that

$$
\left|\xi^{\varepsilon}-\eta^{\varepsilon}\right|^{2} \leq e^{-\min \left\{\rho_{1}, \rho_{2}\right\}(t-\tau)+2 \int_{\tau}^{t} \mathcal{G}_{\delta}\left(\theta_{s} \omega\right) \mathrm{d} s+C\left(\omega, \xi_{\tau}, \eta_{\tau}\right)(t-\tau)^{\frac{1}{3}}}\left|\xi_{\tau}-\eta_{\tau}\right|^{2}
$$

Noticing that $\rho_{1}, \rho_{2}>0$, thus we can obtain

$$
\lim _{t \rightarrow+\infty}\left|\xi^{\varepsilon}(t)-\eta^{\varepsilon}(t)\right|^{2}=0
$$

which implies that the random attractor sets of coupled system (2) are singleton sets.

Remark 3.1 Since $(0,0)$ is the solution of (2), it follows by Theorem 3.1 that the random attractor is actually a singleton set $\{(0,0)\}$. Thus, $(0,0)$ is a globally asymptotically stable equilibrium point.

Theorem 3.2 The solution $\left(u^{\varepsilon}, v^{\varepsilon}\right)$ of coupled system (1) satisfies

$$
\lim _{\varepsilon \rightarrow+\infty}\left\|u^{\varepsilon}(t)-v^{\varepsilon}(t)\right\|^{2}=0
$$

uniformly on any bounded time-interval $\left[T_{1}, T_{2}\right]$ of $\mathbb{R}$.

Proof Let $\xi^{\varepsilon}=\left(u^{\varepsilon}, v^{\varepsilon}\right)^{T}$ be the solution of (1) with the initial datum $\xi_{\tau}=\left(u_{\tau}, v_{\tau}\right)^{T}$, then we have

$$
\begin{aligned}
& \frac{\mathrm{d}}{\mathrm{d} t}\left\|u^{\varepsilon}-v^{\varepsilon}\right\|^{2}+2\left\|\nabla\left(u^{\varepsilon}-v^{\varepsilon}\right)\right\|^{2} \\
& \leq-2 \rho_{1}\left\|u^{\varepsilon}-v^{\varepsilon}\right\|^{2}+2\left|\rho_{2}-\rho_{1}\right|\left\|u^{\varepsilon}-v^{\varepsilon}\right\|^{2}+2\left|\rho_{2}-\rho_{1}\right|\left\|v^{\varepsilon}\right\|^{2} \\
& \quad-2 \operatorname{Re}(1+i \mu)\left\langle\left|u^{\varepsilon}\right|^{2} u^{\varepsilon}-\left|v^{\varepsilon}\right|^{2} v^{\varepsilon}, u^{\varepsilon}-u^{\varepsilon}\right\rangle
\end{aligned}
$$




$$
\begin{aligned}
& -4 \varepsilon\left\|u^{\varepsilon}-v^{\varepsilon}\right\|^{2}+2 \mathcal{G}_{\delta}\left(\theta_{t} \omega\right)\left\|u^{\varepsilon}-v^{\varepsilon}\right\|^{2} \\
\leq & {\left[-2 \rho_{1}+2\left|\rho_{2}-\rho_{1}\right|-4 \varepsilon+2 \mathcal{G}_{\delta}\left(\theta_{t} \omega\right)\right]\left\|u^{\varepsilon}-v^{\varepsilon}\right\|^{2} } \\
& +\min \left\{\frac{\rho_{1}}{2}, 1\right\}\left\|u^{\varepsilon}-v^{\varepsilon}\right\|_{H^{1}(\mathbb{R})}^{2} \\
& +C\left(u^{\varepsilon}, v^{\varepsilon}\right)\left\|u^{\varepsilon}-v^{\varepsilon}\right\|^{2}+2\left|\rho_{2}-\rho_{1}\right|\left\|v^{\varepsilon}\right\|^{2},
\end{aligned}
$$

where

$$
\begin{aligned}
& C\left(u^{\varepsilon}, v^{\varepsilon}\right)=b\left(\left\|u^{\varepsilon}\right\|_{L^{4}(\mathbb{R})}^{\frac{8}{3}}+\left\|v^{\varepsilon}\right\|_{L^{4}(\mathbb{R})}^{\frac{8}{3}}\right), \\
& b=\frac{3}{2^{\frac{1}{3}}}\left(1+2 \sqrt{2} \sqrt{1+\mu^{2}}\right)^{\frac{4}{3}}\left(\min \left\{\frac{\rho_{1}}{2}, 1\right\}\right)^{-\frac{1}{3}} .
\end{aligned}
$$

Therefore, we have

$$
\begin{aligned}
& \frac{\mathrm{d}}{\mathrm{d} t}\left\|u^{\varepsilon}-v^{\varepsilon}\right\|^{2} \\
& \leq {\left[-\rho_{1}+2\left|\rho_{2}-\rho_{1}\right|-4 \varepsilon+C\left(u^{\varepsilon}, v^{\varepsilon}\right)+2 \mathcal{G}_{\delta}\left(\theta_{t} \omega\right)\right]\left\|u^{\varepsilon}-v^{\varepsilon}\right\|^{2} } \\
&+2\left|\rho_{2}-\rho_{1}\right|\left\|v^{\varepsilon}\right\|^{2} .
\end{aligned}
$$

By the Gronwall inequality, one can obtain

$$
\begin{aligned}
& \left\|u^{\varepsilon}(t)-v^{\varepsilon}(t)\right\|^{2} \\
& \leq e^{\int_{\tau}^{t}\left[-\rho_{1}+2\left|\rho_{2}-\rho_{1}\right|-4 \varepsilon+C\left(u^{\varepsilon}, v^{\varepsilon}\right)+2 \mathcal{G}_{\delta}\left(\theta_{s} \omega\right)\right] \mathrm{d} s}\left\|u_{\tau}-v_{\tau}\right\|^{2} \\
& \quad+2\left|\rho_{2}-\rho_{1}\right| \int_{\tau}^{t}\left\|v^{\varepsilon}\right\|^{2} e^{\int_{s}^{t}\left[-\rho_{1}+2\left|\rho_{2}-\rho_{1}\right|-4 \varepsilon+C\left(u^{\varepsilon}, v^{\varepsilon}\right)+2 \mathcal{G}_{\delta}\left(\theta_{r} \omega\right)\right] \mathrm{d} r} \mathrm{~d} s .
\end{aligned}
$$

Similar to (6) and (10) in the proof of Theorem 3.1, we have

$$
\begin{aligned}
&\left\|v^{\varepsilon}(t)\right\|^{2} \leq\left|\xi^{\varepsilon}(t)\right|^{2} \leq e^{2 \int_{\tau}^{t}\left[-\min \left\{\rho_{1}, \rho_{2}\right\}+\mathcal{G}_{\delta}\left(\theta_{s} \omega\right)\right] \mathrm{d} s}\left|\xi_{\tau}\right|^{2} \\
& \leq C\left(T_{1}, T_{2}, \omega, \xi_{\tau}\right), \\
& \int_{\tau}^{t} C\left(u^{\varepsilon}, v^{\varepsilon}\right) \mathrm{d} s \leq 2 b M^{\prime \prime \frac{2}{3}}(\omega)\left|\xi_{\tau}\right|^{\frac{4}{3}}\left(T_{2}-\tau\right)^{\frac{1}{3}}
\end{aligned}
$$

for $t$ on any bounded time-interval $\left[T_{1}, T_{2}\right]$, where

$$
M^{\prime \prime}(\omega)=\int_{\tau}^{T_{2}}\left|\mathcal{G}_{\delta}\left(\theta_{s} \omega\right)\right| e^{2 \int_{\tau}^{s}\left[-\min \left\{\rho_{1}, \rho_{2}\right\}+\mathcal{G}_{\delta}\left(\theta_{r} \omega\right)\right] \mathrm{d} r} \mathrm{~d} s+\frac{1}{2} .
$$

By (11)-(13) and Lemma 2.2, we obtain

$$
\begin{aligned}
& \left\|u^{\varepsilon}(t)-v^{\varepsilon}(t)\right\|^{2} \\
& \leq e^{2 b M^{\prime \prime \frac{2}{3}}(\omega)\left|\xi_{\tau}\right|^{\frac{4}{3}}\left(T_{2}-\tau\right)^{\frac{1}{3}}} e^{\int_{\tau}^{t}\left[-\rho_{1}+2\left|\rho_{2}-\rho_{1}\right|-4 \varepsilon+2 \mathcal{G}_{\delta}\left(\theta_{s} \omega\right)\right] \mathrm{d} s}\left\|u_{\tau}-v_{\tau}\right\|^{2} \\
& \quad+2\left|\rho_{2}-\rho_{1}\right| \int_{\tau}^{t} C\left(T_{1}, T_{2}, \omega, \xi_{\tau}\right) e^{2 b M^{\prime \prime \frac{2}{3}}(\omega)\left|\xi_{\tau}\right|^{\frac{4}{3}}\left(T_{2}-\tau\right)^{\frac{1}{3}}}
\end{aligned}
$$




$$
\begin{aligned}
& \cdot e^{\int_{s}^{t}\left[-\rho_{1}+2\left|\rho_{2}-\rho_{1}\right|-4 \varepsilon+2 \mathcal{G}_{\delta}\left(\theta_{r} \omega\right)\right] \mathrm{d} r} \mathrm{~d} s \\
\leq & M\left(T_{1}, T_{2}, \omega, \xi_{\tau}\right) e^{-4 \varepsilon\left(T_{2}-\tau\right)} .
\end{aligned}
$$

Then we conclude that

$$
\left\|u^{\varepsilon}(t)-v^{\varepsilon}(t)\right\|^{2} \rightarrow 0, \varepsilon \rightarrow+\infty
$$

uniformly for $t$ on any bounded time-interval $\left[T_{1}, T_{2}\right]$.

Remark 3.2 Theorem 3.2 shows that, for any finite time-interval, the interaction between the equations about $u^{\varepsilon}$ and $v^{\varepsilon}$ will make them behave very much alike when the coupling parameter $\varepsilon$ becomes very large, to some extent which is associated with finite-time synchronization $[25,26]$.

\section{Acknowledgements}

The authors would like to thank editors and referees for their very valuable suggestions and comments.

\section{Availability of data and materials}

Data sharing not applicable to this article as no data sets were generated or analyzed during the current study.

\section{Competing interests}

The authors declare that they have no competing interests.

\section{Authors' contributions}

All authors have read and approved the final manuscript.

\section{Publisher's Note}

Springer Nature remains neutral with regard to jurisdictional claims in published maps and institutional affiliations.

Received: 17 August 2020 Accepted: 19 November 2020 Published online: 14 June 2021

\section{References}

1. Pecora, L.M., Carroll, T.L.: Synchronization in chaotic systems. Phys. Rev. Lett. 64, 821-824 (1990)

2. Buck, J.: Synchronous rhythmic flashing of fireflies II. Q. Rev. Biol. 63, 265-289 (1988)

3. Ermentrout, G.B.: Review of Sync: The Emerging Science of Spontaneous Order [Hyperion Press, New York, 2003] by Steve Strogatz. Not. Am. Math. Soc., 51(3), 312-319 (2004)

4. Yang, T., Chua, L.O.: Impulsive stabilization for control and synchronization of chaotic systems: theory and application to secure communication. IEEE Trans. Circuits Syst. I, Fundam. Theory Appl. 44(10), 976-988 (1997)

5. Pikovsky, A., Rosenblum, M., Kurths, J.: Synchronization: A Universal Concept in Nonlinear Sciences. Cambridge University Press, Cambridge (2001)

6. Carvalho, A.N., Rodrigues, H.M., Dłotko, T.: Upper semicontinuity of attractors and synchronization. J. Math. Anal. Appl. 220(1), 13-41 (1998)

7. Rodrigues, H.M.: Abstract methods for synchronization and applications. Appl. Anal. 62(3-4), 263-296 (1996)

8. Afraimovich, V., Rodrigues, H.M.: Uniform dissipativeness and synchronization on nonautonomous equations. In: International Conference on Differential Equations (Lisboa, 1995), pp. 3-17 (1998)

9. Kloeden, P.E.: Synchronization of nonautonomous dynamical systems. Electron. J. Differ. Equ. 2003, 39 (2003)

10. Caraballo, T., Kloeden, P.E.: The persistence of synchronization under environmental noise. Proc. R. Soc. Lond. Ser. A 461(2059), 2257-2267 (2005)

11. Al-azzawi, S., Liu, J., Liu, X.: Convergence rate of synchronization of systems with addictive noise. Discrete Contin. Dyn. Syst., Ser. B 22(2), 227-245 (2017)

12. Chueshov, I., Kloeden, P.E., Yang, M.: Synchronization in coupled stochastic sine-Gordon wave model. Discrete Contin. Dyn. Syst., Ser. B 21(9), 2969-2990 (2016)

13. Caraballo, T., Kloeden, P.E., Neuenkirch, A.: Synchronization of systems with multiplicative noise. Stoch. Dyn. 8(1), 139-154 (2008)

14. Al-azzawi, S., Liu, J., Liu, X.: The synchronization of stochastic differential equations with linear noise. Stoch. Dyn. 18(6), 1850049 (2018)

15. Li, Z., Liu, J.: Synchronization for stochastic differential equations with nonlinear multiplicative noise in the mean square sense. Discrete Contin. Dyn. Syst., Ser. B 24(10), 5709-5736 (2019)

16. Caraballo, T., Chueshov, I., Kloeden, P.E.: Synchronization of a stochastic reaction-diffusion system on a thin two-layer domain. SIAM J. Math. Anal. 38(5), 1489-1507 (2006/07)

17. Acquistapace, P., Terreni, B.: An approach to Ito linear equations in Hilbert spaces by approximation of white noise with coloured noise. Stoch. Anal. Appl. 2(2), 131-186 (1984) 
18. Kłosek-Dygas, M.M., Matkowsky, B.J., Schuss, Z:: Colored noise in dynamical systems. SIAM J. Appl. Math. 48(2), 425-441 (1988)

19. Li, L., Chen, Z:: Asymptotic behavior of non-autonomous random Ginzburg-Landau equation driven by colored noise. Discrete Contin. Dyn. Syst., Ser. B 26(6), 3303-3333 (2021)

20. Lu, H., Zhang, M.: Dynamics of non-autonomous fractional Ginzburg-Landau equations driven by colored noise. Discrete Contin. Dyn. Syst., Ser. B 25(9), 3553-3576 (2020)

21. Shu, J., Li, P., Zhang, J., Liao, O.: Random attractors for the stochastic coupled fractional Ginzburg-Landau equation with additive noise. J. Math. Phys. 56(10), 102702 (2015)

22. Arnold, L.: Random Dynamical Systems. Springer, Berlin (1998)

23. Gu, A., Wang, B.: Asymptotic behavior of random Fitzhugh-Nagumo systems driven by colored noise. Discrete Contin. Dyn. Syst., Ser. B 23(4), 1689-1720 (2018)

24. Gu, A., Wang, B.: Random attractors of Fitzhugh-Nagumo systems driven by colored noise on unbounded domains. Stoch. Dyn. 19(5), 1950035 (2019)

25. Yang, X., Cao, J.: Finite-time stochastic synchronization of complex networks. Appl. Math. Model. 34(11), $3631-3641$ (2010)

26. Liu, X., Chen, T.: Finite-time and fixed-time cluster synchronization with or without pinning control. IEEE Trans. Cybern. 48(1), 240-252 (2018)

\section{Submit your manuscript to a SpringerOpen ${ }^{\circ}$} journal and benefit from:

- Convenient online submission

- Rigorous peer review

Open access: articles freely available online

- High visibility within the field

- Retaining the copyright to your article

Submit your next manuscript at $\gg$ springeropen.com 